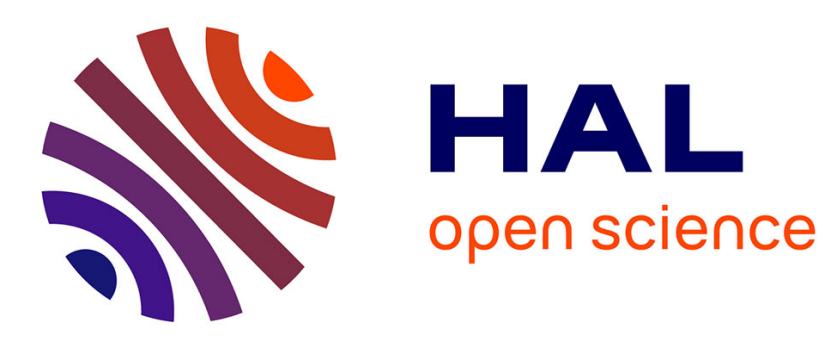

\title{
Chauffage solaire et stratégies de chauffage
}

$\mathrm{X}$. Berger

\section{- To cite this version:}

X. Berger. Chauffage solaire et stratégies de chauffage. Revue de Physique Appliquée, 1981, 16 (5), pp.225-235. 10.1051/rphysap:01981001605022500 . jpa-00244915

\section{HAL Id: jpa-00244915 https://hal.science/jpa-00244915}

Submitted on 1 Jan 1981

HAL is a multi-disciplinary open access archive for the deposit and dissemination of scientific research documents, whether they are published or not. The documents may come from teaching and research institutions in France or abroad, or from public or private research centers.
L'archive ouverte pluridisciplinaire HAL, est destinée au dépôt et à la diffusion de documents scientifiques de niveau recherche, publiés ou non, émanant des établissements d'enseignement et de recherche français ou étrangers, des laboratoires publics ou privés. 


\title{
Chauffage solaire et stratégies de chauffage
}

\author{
X. Berger \\ C.N.R.S., Groupe Ecothermique Solaire, Observatoire de Nice, 06007 Nice, France \\ (Reçu le 29 septembre 1980, révisé le 5 janvier 1981, accepté le 13 février 1981)
}

\begin{abstract}
Résumé. - La répartition des séquences de temps hivernales conditionne l'efficacité d'une installation de chauffage solaire. Si le mode optimal de captage est fonction des types de temps rencontrés, le rôle du stockage est de lisser les apports solaires et d'assurer les périodes critiques. Quant à l'appoint, il doit être le moyen de corriger les conséquences des écarts dans, les successions des journées climatologiques par rapport à un modèle de temps. Le taux de couverture des besoins en chauffage peut atteindre $100 \%$ avec une installation optimisée pour peu qu'une stratégie d'utilisation de l'appoint soit adoptée.
\end{abstract}

\begin{abstract}
The efficiency of a solar heating system depends on the winter time sequence distribution. The types of climatic days determine the adequate collecting mode. The storage function is to smooth the solar gains use and to assume the critical periods. The power supply must be regarded as a way to rectify the deviations in the climatic sequences in regard to a specific model. The heating load can reach $100 \%$ for appropriate installations and by adopting a monitoring strategy.
\end{abstract}

1. Introduction. - L'installation d'un système solaire de chauffage représente un investissement important. Cependant, malgré ce handicap, on observe une diversité de systèmes proposés aux usagers, et une diversité de dimensionnements pour les appareils. Si tout est possible, il existe néanmoins des solutions optimales. Faute de les rechercher, il s'ensuit souvent une déception de l'utilisateur, et cela n'a d'autres conséquences que de desservir les techniques solaires.

Les solutions optimales de chauffage dépendent du climat local, et de l'utilisation prévue pour le bâtiment (en permanence pour un logement, le week-end pour une résidence secondaire, le jour pour des bureaux). L'étude qui suit montre les performances qui peuvent être atteintes par différents systèmes, et fixe les meilleures solutions pour deux régions : Nice et le bassin parisien. Elle se présente comme une suite aux travaux antérieurs menés, tant par nous-même [1], que par d'autres auteurs [2] sur le problème de l'optimisation captage-stockage-appoint.

Les considérations faites pourront paraître simplistes et les calculs effectués manquer de rigueur (par exemple la considération d'un stockage additif et une dégradation brusque au $n+1$-ième jour). Pour être plus rigoureux il aurait fallu prendre en compte différents types d'immeubles (les murs sont eux-mêmes des capteurs !), distinguer les apports directs par les fenêtres des apports par les serres accolées, introduire des coefficients sans dimension pour caractériser les effets couplés température-insolation-hygrométrie, considérer l'inertie des bâtiments, les types de capteurs et de stockage... Finalement on aurait alourdi l'étude qui se veut une analyse synthétique des possibilités et stratégies de chauffage solaire pour montrer comment le climat et les séquences de temps interviennent sur le taux de couverture des besoins en chauffage qui peut être atteint, et établir les dimensionnements optimaux captage-stockage appoint. La considération d'une installation trop particulière ne représenterait qu'un exemple isolé, celle d'un jeu d'installations nécessiterait un livre et ne ferait pas complètement le tour du problème. Or il s'agit non pas de retenir les chiffres présentés et qui peuvent évidemment varier d'une installation à l'autre, mais la philosophie de l'enseignement qui est donnée. Le but recherché est de montrer comment un système peut être, ou non, bien adapté à un climat, et comment on parvient à l'optimiser.

2. Présentation de l'étude. - Une installation est dimensionnée proportionnellement au volume $V$ et au coefficient $G$ de déperditions volumiques de l'habitat, ainsi qu'en fonction de l'usage qui en est fait. C'est pourquoi, l'unité adoptée pour mesurer l'importance des systèmes de captage ou de stockage a été choisie pour faire référence au bâtiment et non à des $\mathrm{m}^{2}$ ou des $\mathrm{m}^{3}$; en définissant "cs " comme la quantité d'énergie nécessaire à la satisfaction des besoins de 
chauffage du bâtiment au 15 janvier par 8,50 de température moyenne de l'air extérieur, et en ignorant les apports solaires, on s'affranchit de la définition ou de la marque d'un type d'appareils, et on classe dans une même famille tous les systèmes ayant les mêmes performances. Cette définition inclut l'énergie secondaire (électricité, ...) nécessaire au fonctionnement des capteurs ou à la restitution du stockage; il y a donc lieu de corriger les résultats annoncés en fonction de l'énergie secondaire dépensée.

Pour ne pas alourdir la compréhension du raisonnement, il a été admis que les performances des systèmes de captage et de stockage étaient additives, ce qui est presque vrai pour un stockage par chaleur latente de fusion, type de stockage dont l'intérêt s'affirme de jour en jour, ou pour les systèmes classiques, lorsque les températures des fluides ne s'écartent pas de certaines valeurs moyennes. Pour les stockages à $n$ jours (c'est-à-dire ne pouvant se conserver plus de $n$ jours), il a été admis par simplification la dégradation complète au $n+1$ jour.

Pour le captage, l'énergie récupérée varie de $10 \%$ par degré de température moyenne extérieure, et est proportionnelle au rayonnement incident journalier. Cela permet de tenir compte raisonnablement des performances des divers systèmes de captage qui co-existent dans une installation solaire (capteurs classiques, apports directs, murs Trombe, serres accolées...), des seuils de fonctionnement et des surchauffes, des pertes par transmissions, ... Différentes études fines de comportements d'immeubles réels ont permis de s'arrêter sur ce taux de $10 \%$ et sur cette manière de chiffrer l'énergie captée, mais il est tout à fait possible, vue la large dispersion qui existe dans le comportement des capteurs, de rencontrer des systèmes $20 \%$ plus ou moins efficaces. Par ailleurs, on considère des tranches de $24 \mathrm{~h}$, et on suppose donc que l'énergie captée peut contribuer pendant $24 \mathrm{~h}$ à la satisfaction des besoins. Si cet étalement ne peút être naturel (inertie), il faudra compter un stockage supplémentaire d'environ 0,5 «cs » et de durée 1 jour. La considération d'un stockage annuel a paru intéressante pour plusieurs raisons :

- lorsqu'il n'est utilisé qu'une fois, il équivaut à un combustible non renouvelable (bois, électricité...) et il

1
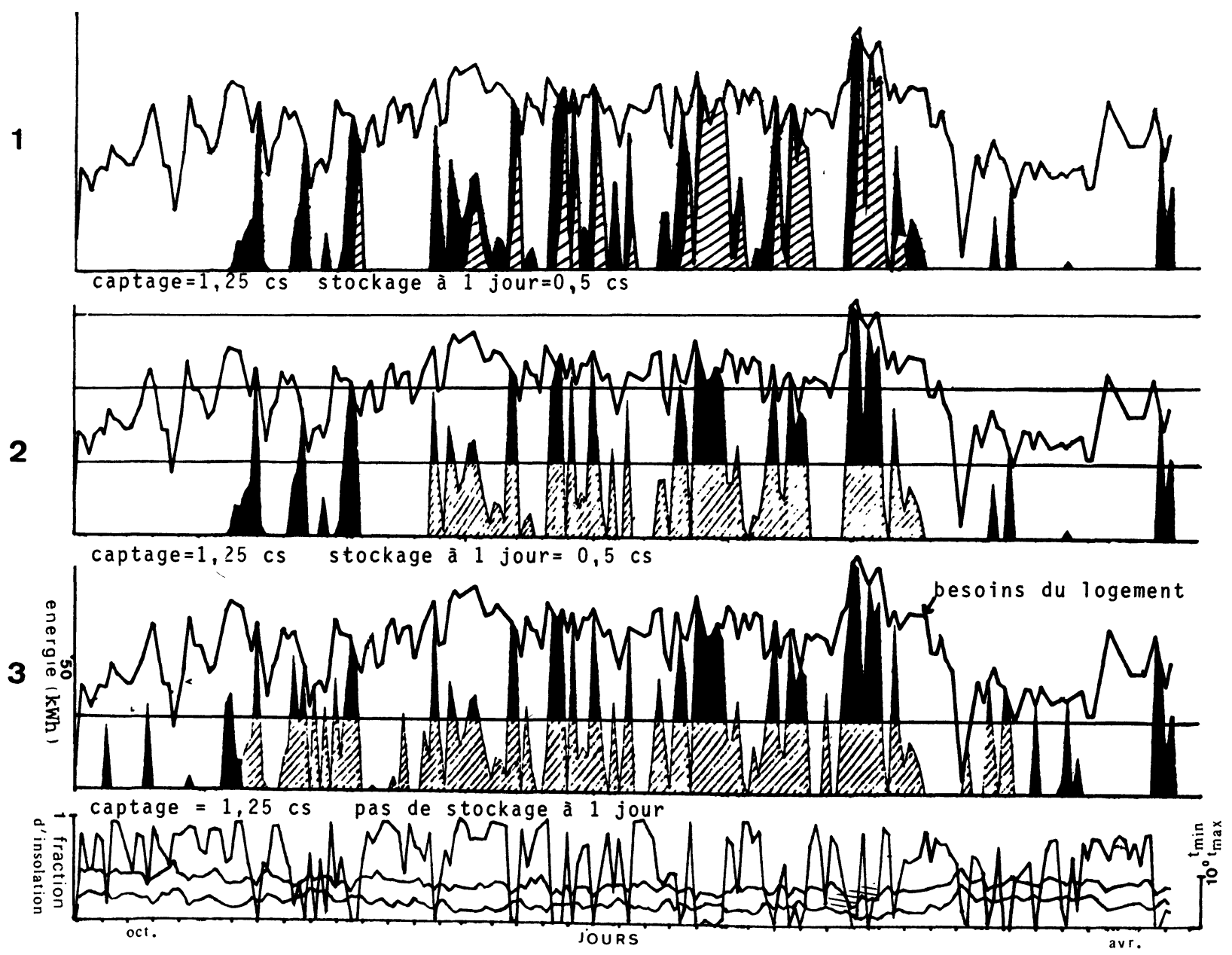

Fig. 1. - Utilisation du chauffage d’appoint (Nice 1973-74) : $1:$ après utilisation du stockage ; 2 et 3 : en même temps que le stockagé pour en limiter la puissance. besoins devant être assurés par le stockage;

[Set-up power use (Nice 1973-74) : $1:$ when the storage is empty ; 2 and $3:$ in parallel with the storage to limit the set-up power. load to be assumed by the storage ; $/$ heating load to be assumed by the set-up power.] 
permet donc une évaluation de l'appoint nécessaire;

- les recherches en cours sont en train d'aboutir à la mise au point d'un tel stockage, et on peut espérer une commercialisation d'ici quelques années.

De même la considération de capteurs fonctionnant à basse température en association avec une pompe à chaleur a paru aussi intéressante pour des comparaisons avec les capteurs dits classiques pour lesquels les calories produites le sont à un niveau de température supérieur à celui qui est utilisé à l'intérieur de l'habitat.

Sur ces bases, les besoins en chauffage d'un logement ont été calculés jour par jour pour un hiver réel décrit à partir des données fournies par la Météorologie Nationale [3]; on a aussi calculé l'énergie récupérée par le système de captage, l'état du stockage et la couverture des besoins. Ces calculs ont été effectués sur ordinateur pour une variété de systèmes et de stratégies d'utilisation depuis le premier novembre jusqu'au premier mai.

3. Considérations météorologiques. - En hiver, le sud-est de la France est partagé entre deux types de temps : le ciel clair (48\% des journées), et le ciel couvert (Fig. 1), l'alternance des deux types de temps étant assez régulière (Fig. 2). Les journées de beau temps sont groupées par deux ou trois, celles de

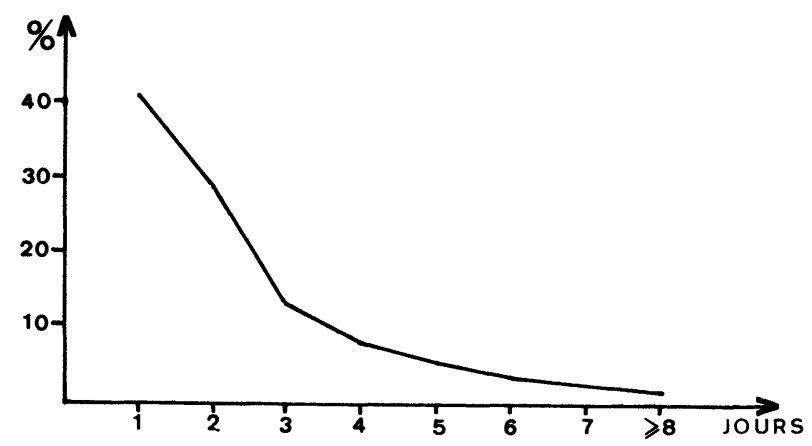

Fig. 2. - Probabilité d'avoir $n$ jours de mauvais temps après 1 ou 2 jours de beau temps (Nice - Données de la Météorologie Nationale - moyenne des hivers 74-75, 75-76, 76-77).

[Probability (in \%), of getting $n$ cloudy days after 1 or 2 clear days (Nice - National Meteorological data - winters 74-75, 75-76, 76-77).]

mauvais temps par deux au plus dans la majorité des cas (Fig. 3). Les longues séquences de mauvais temps ont lieu le plus souvent en milieu ( 3,3 fois plus) ou fin $\mathrm{d}$ 'hiver (1,9 fois plus) qu'en début d'hiver. Les longues séquences de beau temps se trouvent plus généralement en début (1,3 fois plus) ou milieu ( 1,4 fois plus) d'hiver qu'en fin d'hiver. Bien que les années ne se ressemblent pas toutes pour le temps, ces caractéristiques des séquences de temps demeurent assez constantes : une longue période de beau temps entre la minovembre et la fin janvier, encadrée par deux périodes pluvieuses qui se situent après l'équinoxe d'automne et avant celui de printemps. Les micro-climats très

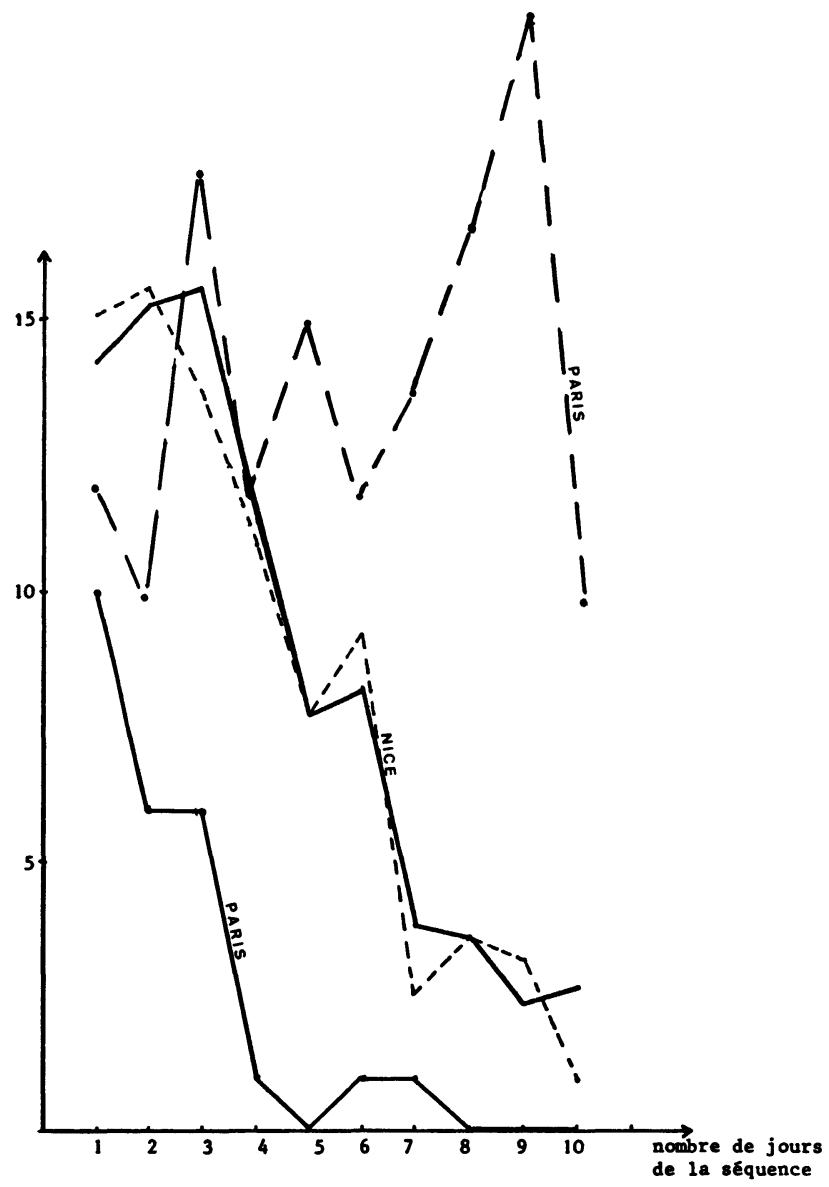

Fig. 3. - Durée moyenne des séquences de temps pendant le semestre hivernal (15 octobre au 15 avril). $N$ : nombre de jours d'hiver appartenant à une séquence de durée donnée (données de la Météorologie Nationale; moyenne sur 11 années). — séquences de beau temps; ...- séquences de mauvais temps.

[Histogram of clear ( $\longrightarrow$ ) and cloudy (- - ) sequences during winter period (National Meteorological data for Nice during 11 years).]

marqués résultent de la présence de la mer et de la montagne.

La température moyenne de l'air est supérieure d'environ $3^{\circ}$ par mauvais temps à celle qu'elle a par beau temps.

La région parisienne est marquée l'hiver par un temps généralement couvert $(10 \%$ de jours de beau temps) et les températures d'air y sont plus froides qu'à Nice; l'hygrométrie y est moins élevée.

Ces caractéristiques sont les principales à considérer pour une compréhension de l'efficacité d'un système. D'autres détails [3] seraient aussi à considérer pour l'établissement de stratégies de chauffage très élaborées que nous ne présenterons pas ici.

De ces éléments du climat ressortent les critères principaux pour le choix et le calcul d'une installation :

- en région niçoise, l'importance du captage (capteurs fonctionnant par beau temps et non par mauvais temps : capteurs notés de type 1) est marquée, tant par le pourcentage de jours de beau temps que par l'alternance régulière des jours de beau et mauvais 
temps, du fait aussi de la présence d'une longue période de beau temps au solstice, c'est-à-dire quand le soleil est au plus bas sur l'horizon. De même l'intérêt d'un stockage pour une à deux journées est mis en évidence [1],

- en région parisienne c'est la faible efficacité hivernale de ce type de capteurs qui est soulignée. Les solutions sont à rechercher sous la forme de grandes surfaces de serres accolées, vérandas ou loggias vitrées qui jouent alternativement les rôles de capteurs, stockage, espaces tampons et zones partiellement habitables [6]. Dans de tels volumes, une partie de l'énergie captée est stockée sur place pour assurer le confort, l'autre partie est transmise directement au logement par une circulation d'air intermittente, ou indirectement par l'intermédiaire d'une pompe à chaleur, d'un échangeur à plaques et (ou) d'un stockage. Ces solutions sont plus difficilement contrôlables en région niçoise, bien qu'évidemment efficaces [4, 5].

4. Interprétation des résultats pour la région niçoise et pour les capteurs de type 1. - La figure 4 représente les résultats obtenus par ordinateur. On y retrouve fortement marquée l'importance du captage : en dessous d'un certain seuil le rôle du stockage est négligeable car l'énergie captée ne suffit pas aux besoins, sauf parfois en début ou fin d'hiver.

$\mathrm{Si}$ le stockage est faible, peu importe son type. journalier ou annuel; l'explication provient du fait que $41 \%$ des jours de mauvais temps sont précédés par des jours de beau temps (Fig. 2); le stockage annuel, étant fréquemment vidé, jusqu'à 17 fois dans l'hiver (Fig. 4), ne se différencie pas quant à son type. d'autant plus qu'il ne peut satisfaire une demande pendant plusieurs jours de suite.

Si la première demi-journée $(1 / 2 \mathrm{cs})$ de stockage annuel peut être déchargée et chargée jusqu'à 17 fois pendant l'hiver, la journée suivante ne l'est plus que 9 fois, celle après 5 fois, la troisième 3 fois, et les suivantes 1 fois ; ainsi, si avec un captage de 2 cs et un stockage annuel de 4 cs on peut assurer $93 \%$ des besoins, il reste quand même 14 jours de temps froid et couvert non pourvus; il faut donc $14 \mathrm{cs}$ de stockage annuel en plus pour atteindre les $100 \%$. L'intérêt d'un tel stockage, ne cyclant qu'une fois par an, peut se poser, vu son coût et son encombrement (stockage rempli sans problème en été). Etant donné son équivalence avec tout combustible non renouvelable (bois, charbon...) lorsque le cycle n'est que de 1 par an, il est facile de déterminer l'appoint optimal.

Le climat niçois incite l'usager à posséder plutôt un système de captage important qu'un ensemble de stockage. Pourtant les investissements ne sont pas les mêmes; de plus, l'esthétique de l'habitat est plus difficile à maintenir avec des capteurs qu'avec un stockage. Enfin l'inutilité des capteurs pendant la moitié de l'année, et la possibilité de remplir pendant l'été un stockage annuel, incitent plutôt l'utilisateur à posséder le contraire ; il y a donc un choix à effectuer :

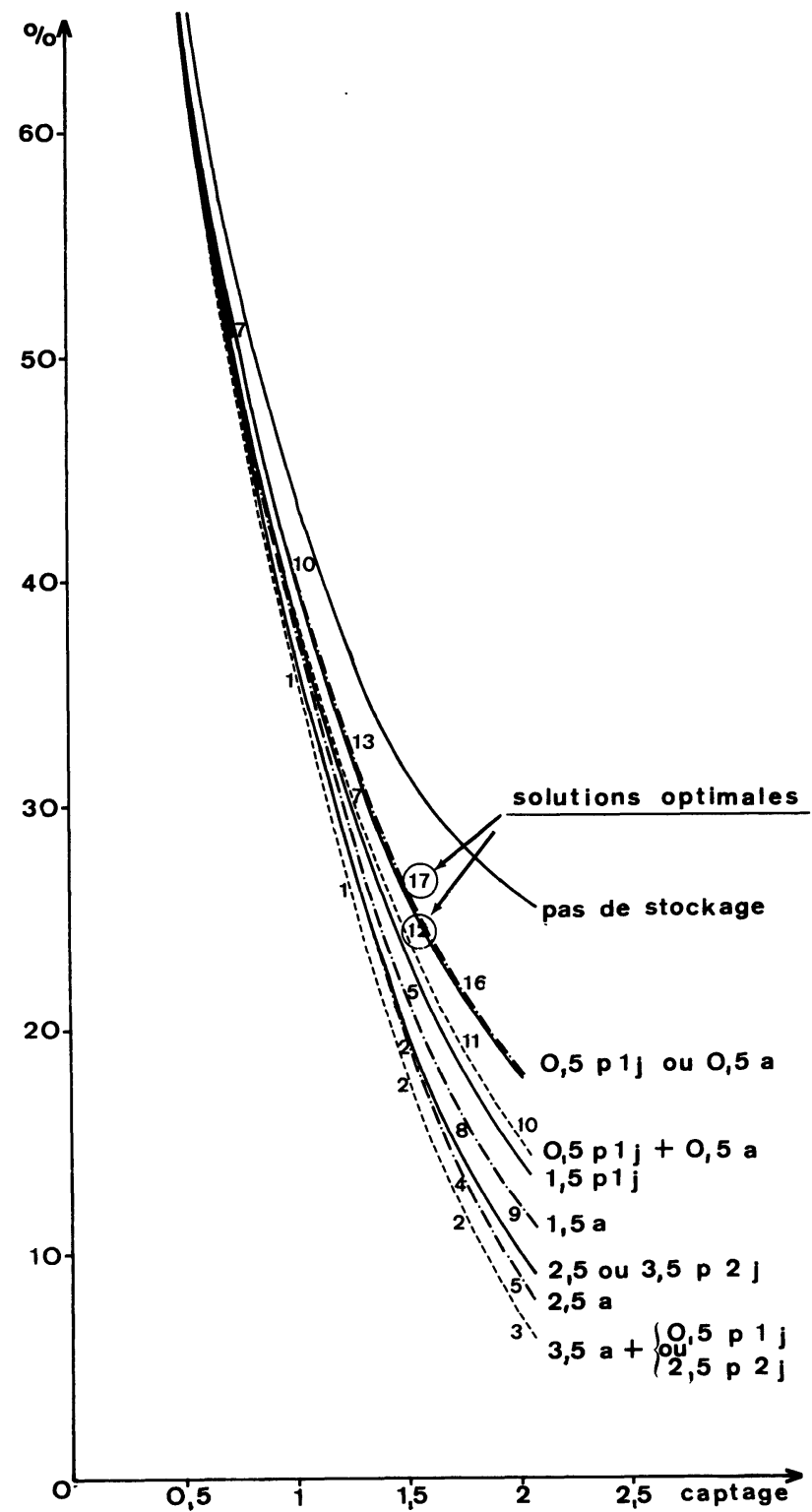

Fig. 4. - Nice - capteur 1. Pourcentage des besoins non couverts en fonction de l'importance du captage et du stockage (journalier ou annuel) exprimés en unités "cs »; les chiffres le long des courbes indiquent le nombre de fois où le stockage a été complètement vidé, puis complètement rempli.

[Nice-Classical collector. Percentage of heat requirements not covered by solar gains as a function of collection and storage (daily $\mathrm{j}$, annual a) expressed in "cs " units. The numbers along the curves express the number of times when the storage has been emptied and filled again.]

Pour la seule considération de l'optimisation technique captage-stockage, la taille du stockage qui permet, pour un captage donné, de le remplir et de le vider complètement le plus de fois possible est la meilleure. On a cette solution pour l'une des combinaisons (Fig. 4) :

$$
\begin{gathered}
\text { captage }=1,5 \mathrm{cs} \text { stockage }=0,5 \mathrm{plj} \text { ou } \\
0,5 \mathrm{a} \Rightarrow 74 \% \text { des besoins satisfaits } \\
\text { captage }=1,5 \mathrm{cs} \quad \text { stockage }=0,5 \mathrm{plj}+ \\
0,5 \mathrm{a} \Rightarrow 76 \% \text { des besoins satisfaits } .
\end{gathered}
$$


Pour parvenir à un captage journalier important, l'effort doit être porté dans plusieurs directions :

- diminuer les déperditions volumiques, c'est augmenter "cs »

- essayer d'assurer les besoins du logement par jours de beau temps (jour + nuit) par des apports directs (fenêtres) ou différés (serres accolées, mezzanines, murs Trombe ou diode), et par inertie.

Ces efforts permettent de limiter la surface des capteurs externes à la maison, c'est-à-dire contribuent à adapter l'habitat à sa région et à son climat. C'est d'ailleurs par ces actions sur l'habitat que l'on rapprochera l'optimum financier de l'optimum technique.

5. Utilisation d'un chauffage d'appoint. - En partant partant de la conclusion qu'un chauffage d'appoint est nécessaire, la question se pose de la dimension qu'il faut lui donner, de la manière de s'en servir, et de ce que devient alors le chauffage solaire.

En région parisienne, le chauffage solaire ne peut satisfaire qu'une faible part des besoins. C'est pourquoi le chauffage complémentaire ne peut être appelé chauffage d'appoint : il ne peut en effet qu'être dimensionné aux besoins maxima du logement.

En région niçoise il n'en est pas de même, étant donné le grand nombre de jours de beau temps et la constitution des séquences de temps.

Si le captage est trop faible, l'intérêt du stockage est nul, et le chauffage d'appoint ne peut avoir d'autre rôle que de compléter le captage par grand froid ou mauvais temps. Il doit donc être dimensionné au maximum des besoins de l'habitation. Par contre, si le captage est suffisant pour assurer la plupart du temps un peu plus que les besoins par beau temps, et s'il y a un stockage non négligeable, il existe un dimensionnement optimal du chauffage d'appoint et une stratégie d'utilisation :

La méthode consistant à utiliser d'abord le stockage annuel, puis l'appoint dès que les besoins ne sont plus satisfaits par l'ensemble captage-stockage journalier n'est pas idéale dans la mesure où elle nécessite pour dimensionnement de l'appoint la satisfaction des besoins maximaux (Fig. 1). Cependant, en début ou fin de saison froide, elle reste bonne : en effet les besoins étant réduits et les apports par beau temps importants, il y a d'une part une probabilité minime d'avoir besoin de l'appoint, d'autre part le stockage peut assurer plus de jours de chauffage qu'en milieu d'hiver; enfin on est assuré de pouvoir reconstituer très vite le stockage.

Si le stockage ne peut se conserver qu'une ou deux journées, il faut garder cette stratégie en milieu d'hiver et l'appoint doit donc rester égal au maximum des besoins : en effet il y a en moyenne en hiver 37 jours de mauvais temps pour 53 de beau après un jour de beau temps, soit $41 \%$ de chances d'avoir besoin du stockage (Fig. 2). Par ailleurs il y a environ 60 jours de mauvais temps qui suivent une journée de mauvais temps et 32 qui en suivent 2 ; il existe donc un trop grand nombre de journées où seul l'appoint peut assurer le chauffage. Avec un dimensionnement au maximum des besoins il n'y a pas d'intérêt à effectuer de stratégie particulière.

S'il existe un stockage annuel, il y a intérêt à le maintenir chargé en milieu d'hiver, car il sera toujours temps de l'utiliser en fin d'hiver. Mais peut-on compter sur le captage ? Un captage de 1 cs ne permet de recharger que 6 fois la première demi-journée de stockage, alors qu'un captage de 1,5 cs le permet 12 fois, ce qui est presque le maximum utile (15 fois). L'appoint doit donc remplacer un peu plus que $0,5 \mathrm{cs}$ et assurer les 9 fois nécessaires. Soit un appoint égal à $0,5 \mathrm{cs}$ :

Du fait que l'on a moins d'une chance sur 2 d'avoir besoin du stockage le lendemain, on n'utilisera donc cet appoint qu'une fois sur 2 par beau temps lorsque le stockage sera vide; ainsi le captage pourra recharger le stockage. Dans ces conditions, l'appoint ne remplacera que 5 fois sur 9 les 0,5 cs de captage. Si le stockage était limité à $0,5 \mathrm{cs}$, il resterait près de 60 jours où l'appoint assurerait seul le chauffage, et la couverture des besoins serait loin d'être satisfaite à $100 \%$.

Avec un stockage de $2 \mathrm{cs}$, il ne reste que 5,2\% de journées qui ne sont satisfaites que par l'appoint, soit 4,8\% des besoins qui ne sont pas assurés. Avec un stockage de 3 cs il n'y a plus que 1,9\% des besoins non assurés, soit 3,5 jours.

Etant donné que, sur la distribution des couples de jours qui suivent un jour de beau temps, il y a $29 \%$ de groupes de 2 jours de mauvais temps, on ne remplira la deuxième demi-journée de stockage qu'une fois sur 3 lorsqu'il fera beau et que la première demijournée sera remplie. Pour les autres demi-journées on n'utilisera pas l'appoint pour les remplir. C'est dans ces conditions que l'on utilisera au mieux l'appoint.

Si l'on tient à assurer la totalité des besoins, il faut malheureusement assurer les $1,9 \%$ des besoins restants et donc avoir en plus 3,5 cs de stockage. Ceux-ci ne seront utilisés qu'une fois dans l'hiver et non rechargés avant l'été.

En résumé les résultats sont les suivants :

- appoint $=1 \quad$ cs : pas de stratégie,

- appoint $=0,33 \mathrm{cs}$ : appoint faible; il faut 2 jours de beau temps pour remplir 1 jour d'utilisation du stock; or il y a $20,7 \%$ de chances d'avoir 2 jours de beau temps après un jour de beau suivi d'un jour couvert; il y a donc obligation de remplir le stockage chaque fois que c'est possible; d'où un gâchis d'énergie solaire let un appoint trop utilisé; - appoint $0,5 \mathrm{cs}$, captage $=1 \mathrm{cs}$, stockage $=6 \mathrm{cs}$. L'appoint assure $37 \%$ des besoins avec la stratégie et non $34 \%$ (utilisation maximale de l'énergie solaire), et tous les besoins sont assurés. Sans stratégie, l'appoint assure $31 \%$, mais il reste $3 \%$ des besoins qui ne sont pas satisfaits et qui ne peuvent l'être par l'appoint ;

- appoint $0,5 \mathrm{cs}$, captage $=1,5 \mathrm{cs}$, stockage $=6 \mathrm{cs}$.

L'appoint assure $17,5 \%$ des besoins et non $12 \%$; sans stratégie il assure $15,5 \%$ des besoins, mais il 
reste $1,5 \%$ des besoins qui ne sont pas satisfaits (environ 6 journées où la couverture des besoins n'est qu'à moitié assurée) ;

- captage 1,7 cs : l'intérêt de l'appoint tombe car il n'est pas assez utilisé ; de même il y a trop de captage et l'installation est trop onéreuse.

L'appoint se présente ainsi non seulement comme un complément du solaire, mais aussi comme un moyen d'optimiser chacune des trois parties captagestockage-appoint. Il existe un barycentre de performances, et un autre d'économies, entre les tailles de ces trois éléments. Sur la figure 5 sont positionnés les barycentres $\mathrm{G}$ et $\mathrm{G}^{\prime}$ pour des couvertures de besoins de $74 \%$ et $90 \%$. Les points d'égales performances se situent sur une surface presque plane, d'incurvation variable tournée vers l'origine des axes; l'inclinaison moyenne de cette surface relativement au plan cap- tage-stockage varie avec l'indice de performance, ce qui fait déplacer le barycentre plus rapidement vers les zones de fort stockage et fort appoint que vers celle de fort captage lorsque croît le pourcentage de couverture des besoins. Cette figure montre comment on passe d'une installation surdimensionnée, et pour laquelle le seul critère retenu est la couverture maximale des besoins par l'énergie solaire, à une installation optimisée pour laquelle on souhaite l'investissement le plus raisonnable pour une énergie d'appoint minimale.

\section{Interprétation des résultats pour la région niçoise} et les capteurs de type 2 . - En plus des capteurs, dits classiques, et ne fonctionnant pratiquement que par beau temps, il existe des systèmes qui permettent la récupération de calories, même par mauvais temps;

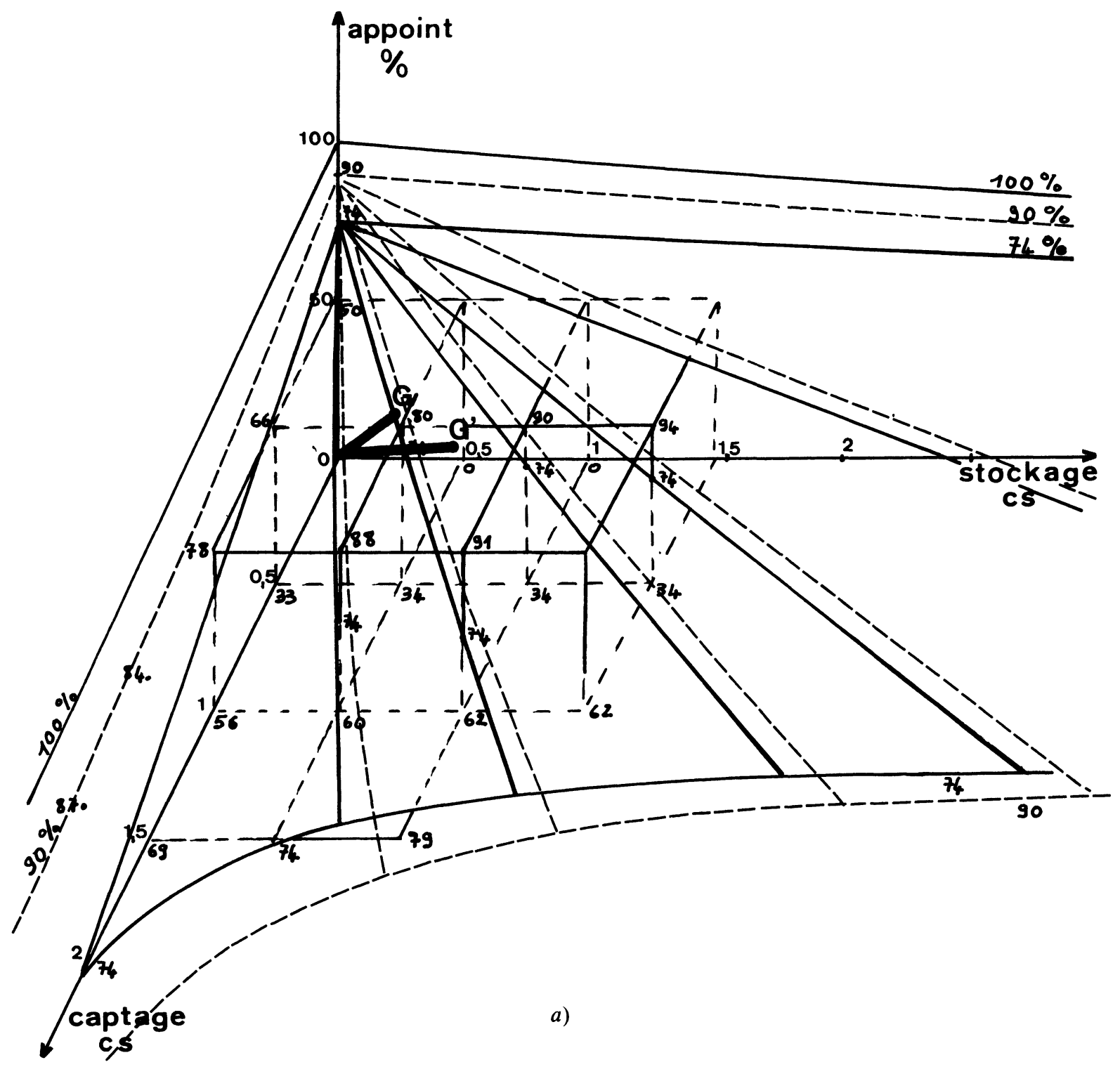

Fig. 5a. - Optimisation captage-stockage-appoint (Nice - capteur 1). Surfaces d'égale couverture des besoins ( $74 \%$ et $90 \%$ ). G, G' : solutions optimales (distances minimales à l'origine).

[Optimization collecting-storage-set-up power (Nice - classical collectors). Areas of equal satisfied heating loads ( $74 \%$ and $90 \%$ ). G and $\mathrm{G}^{\prime}$ : the best solutions (minimal distances to the origin point).] 


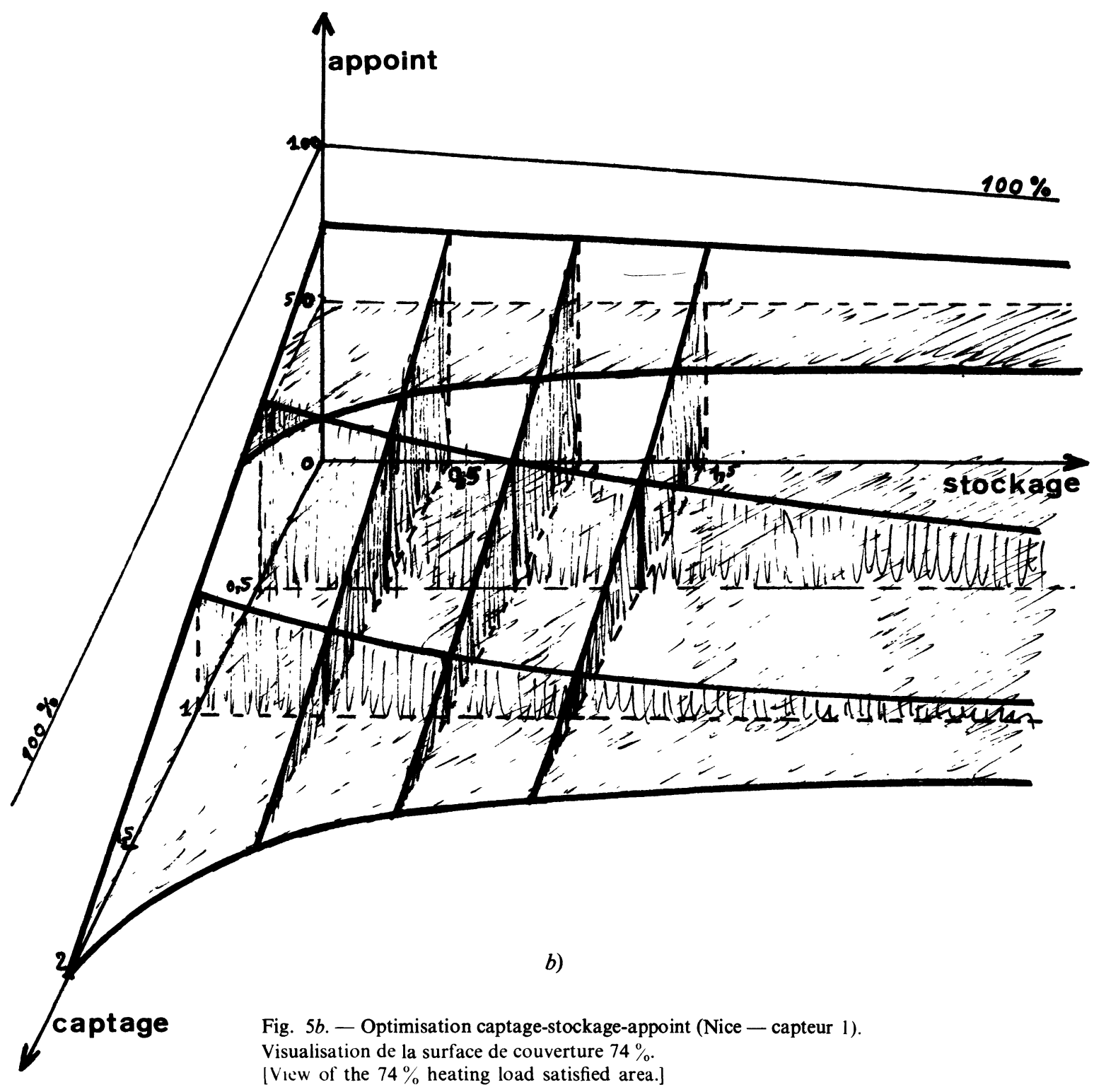

leur principe est une association avec une pompe à chaleur de manière à fonctionner avec un fluide très froid; il y a ainsi récupération de l'énergie de condensation de la vapeur d'eau contenue dans l'air [7]. L'intérêt de ces capteurs, notés de type 2, est de fonctionner par tous les temps et de réduire par là la nécessité de déphasage temporel de calories (pertes + déperditions); en région niçoise où le degré hygrométrique de l'air est élevé, et la température moyenne de l'air plus élevée par mauvais temps que par beau temps, on peut obtenir par mauvais temps 40 à $60 \%$ de l'énergie récupérable par beau temps (Fig. 6). Nous avons adopté $40 \%$ dans l'étude effectuée ici. Enfin il ne faut pas oublier que ces capteurs nécessitent une énergie secondaire importante (entre 15 et $30 \%$ de l'énergie captée), et il y a donc lieu de faire intervenir cette considération dans les résultats donnés par les figures 7 et 8 pour déterminer la couverture des besoins par les apports solaires.

Les résultats obtenus accentuent le rôle du captage

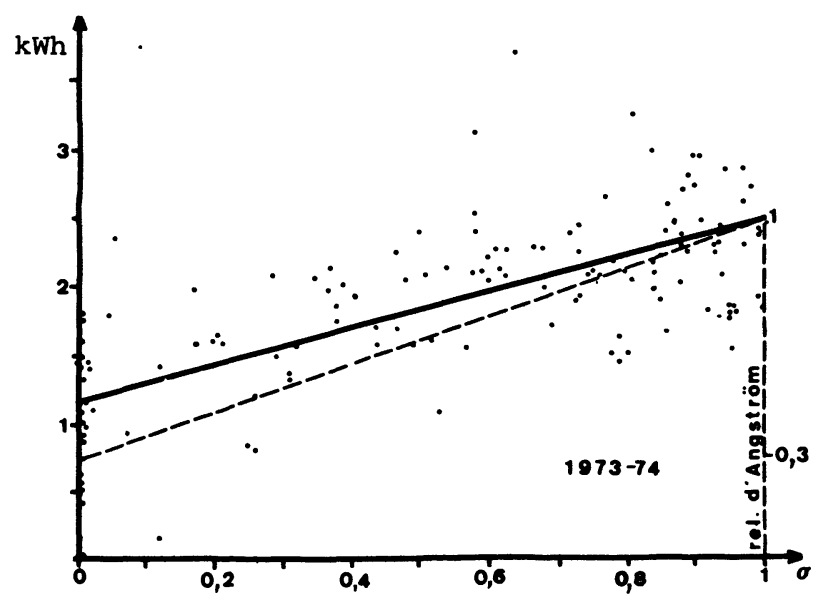

Fig. 6. - Energie solaire captée par jour et par mètre carré en fonction de la fraction d'insolation (Nice, $1^{\mathrm{er}}$ novembre au 15 mars), capteurs de type 2. Simulations pour une maison à Juan-les-Pins.

[Solar energy collected by day and by meter square versus the insolation fraction (Nice - 1st Nov. to 15th Mar.). Collectors of type 2. Simulations for a house situated in Juan-les-Pins, near Nice.] 
et présentent le stockage comme un lissage des apports des jours de beau temps sur ceux de mauvais temps.

Le stockage annuel perd son intérêt puisque la première demi-journée n'est au mieux chargée et déchargée que 8 fois au lieu de 17. Les solutions optimales obtenues sont :

$$
\left.\begin{array}{rl}
\text { captage } & =1,5 \mathrm{cs} \\
\text { stockage } & =0,5 \mathrm{plj}+0,5 \mathrm{a} \\
\text { captage } & =1,75 \mathrm{cs} \\
\text { stockage } & =0,5 \mathrm{p} 1 \mathrm{j} \text { ou } 0,5 \mathrm{a} \\
\text { captage } & =1,75 \\
\text { stockage } & =2,5 \mathrm{p} 2 \mathrm{j}+0,5 \mathrm{a} \text { ou } \\
3 \mathrm{a}
\end{array}\right\}
$$

$88 \%$ des besoins satisfaits

$92 \%$ des besoins satisfaits

$96 \%$ des besoins satisfaits

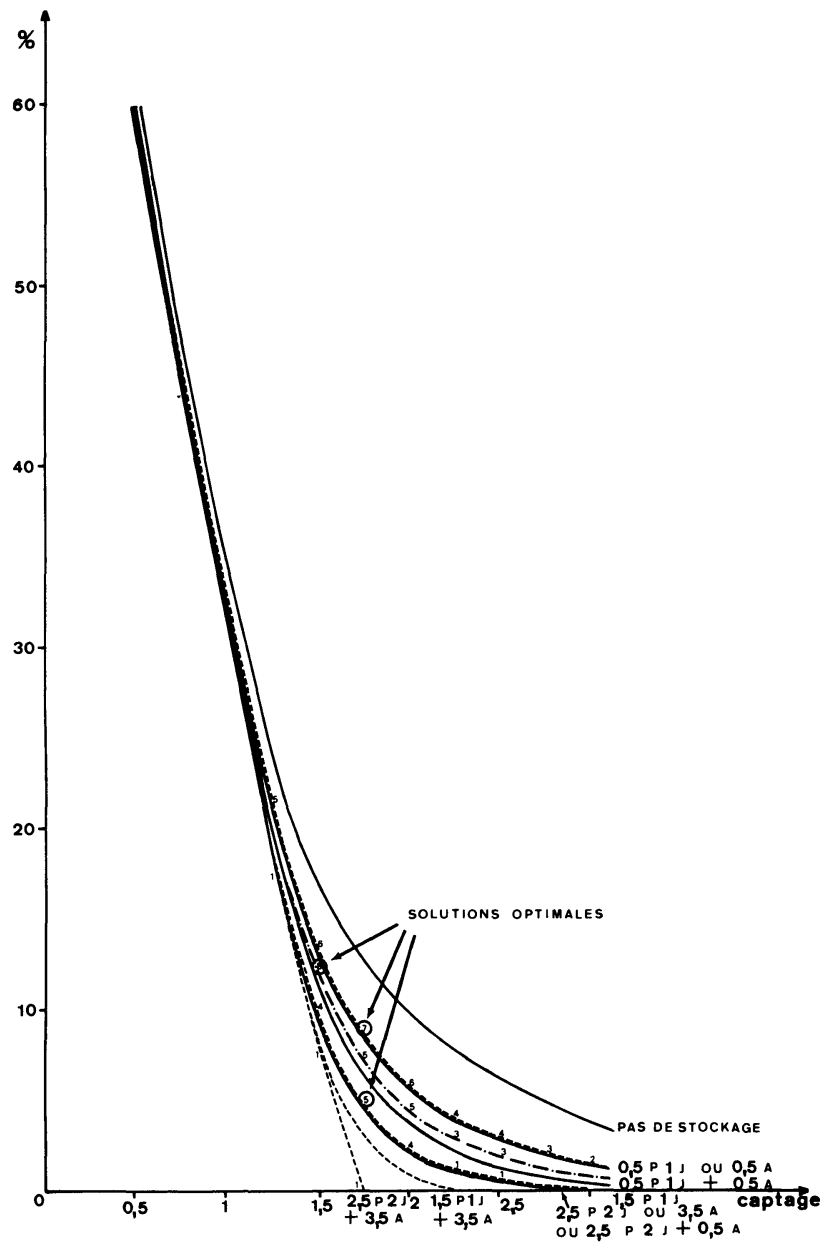

Fig. 7. - Nice - capteur 2.

[Nice - collectors of type 2 (drawing conventions same as in Fig. 4).]

Ces résultats, si bons soient-ils, sont cependant à peine meilleurs que ceux obtenus avec les capteurs de type 1 :

Considérons en effet les deux cas suivants :

$$
\text { captage }=1,5 \mathrm{cs} \quad \text { stockage }=0 \mathrm{cs}
$$

énergie secondaire pour le capteur $1=5 \%$ énergie secondaire pour le capteur $2=20 \%$.
Pour le capteur I la couverture solaire est de $65 \%$; elle est de $68 \%$ pour le capteur 2 .

Ce résultat montre que ce type de capteur ne doit être utilisé qu'avec une pompe à fort $\mathrm{COP}$ et en région humide (en région niçoise le taux d'énergie récupérable par mauvais temps peut atteindre $60 \%$ et non seulement $40 \%$ comme il a été considéré), ou bien en région humide et à ciel fréquemment couvert (Vendée, Bretagne).

Du point de vue financier le capteur à condensations peut être très bon marché même compte tenu de l'investissement pompe ì chaleur. C'est pourquoi il conserve son intérêt.

7. Interprétation des résultats pour la région parisienne. - 7.1 CAPTEURS DE TYPE 1. - Les courbes de la figure 8 montrent la faible efficacité du captage et le peu d'intérêt d'un stockage : au mieux peut-on vider et remplir 3 fois dans l'hiver un stockage d' $1 / 2$ cs. C'est pourquoi, dans une telle région l'effort doit être porté sur l'isolation et les systèmes à fonctions multiples : les risques de surchauffes sont faibles et une gestion naturelle simple peut être aisément obtenue ; de plus le coût d'investissement est alors mieux rentabilisé.

7.2 Capteurs de type 2. - Ce type de capteur est mieux adapté que le précédent au bassin parisien; en effet, même si l'hygrométrie de la région est moins forte qu'en région niçoise, le nombre de jours de temps médiocre compense cet inconvénient. En reprenant l'exemple précédent, on obtient pour la couverture des besoins par l'énergie solaire : $16 \%$ contre $17 \%$ pour les capteurs de type 1. C'est donc bien vers les systèmes qui ont une efficacité par mauvais temps qu'il convient de s'orienter et c'est bien le rôle des espaces survitrés accolés : à défaut de ne pouvoir être à ces moments des capteurs, ils se comportent alors comme des espaces-tampons et de plus leur habitabilité, même intermittente [6] est appréciable.

8. Cas des résidences secondaires utilisées seulement le week-end. - Le cas des bureaux utilisés uniquement le jour s'identifie à celui des habitations ordi- 


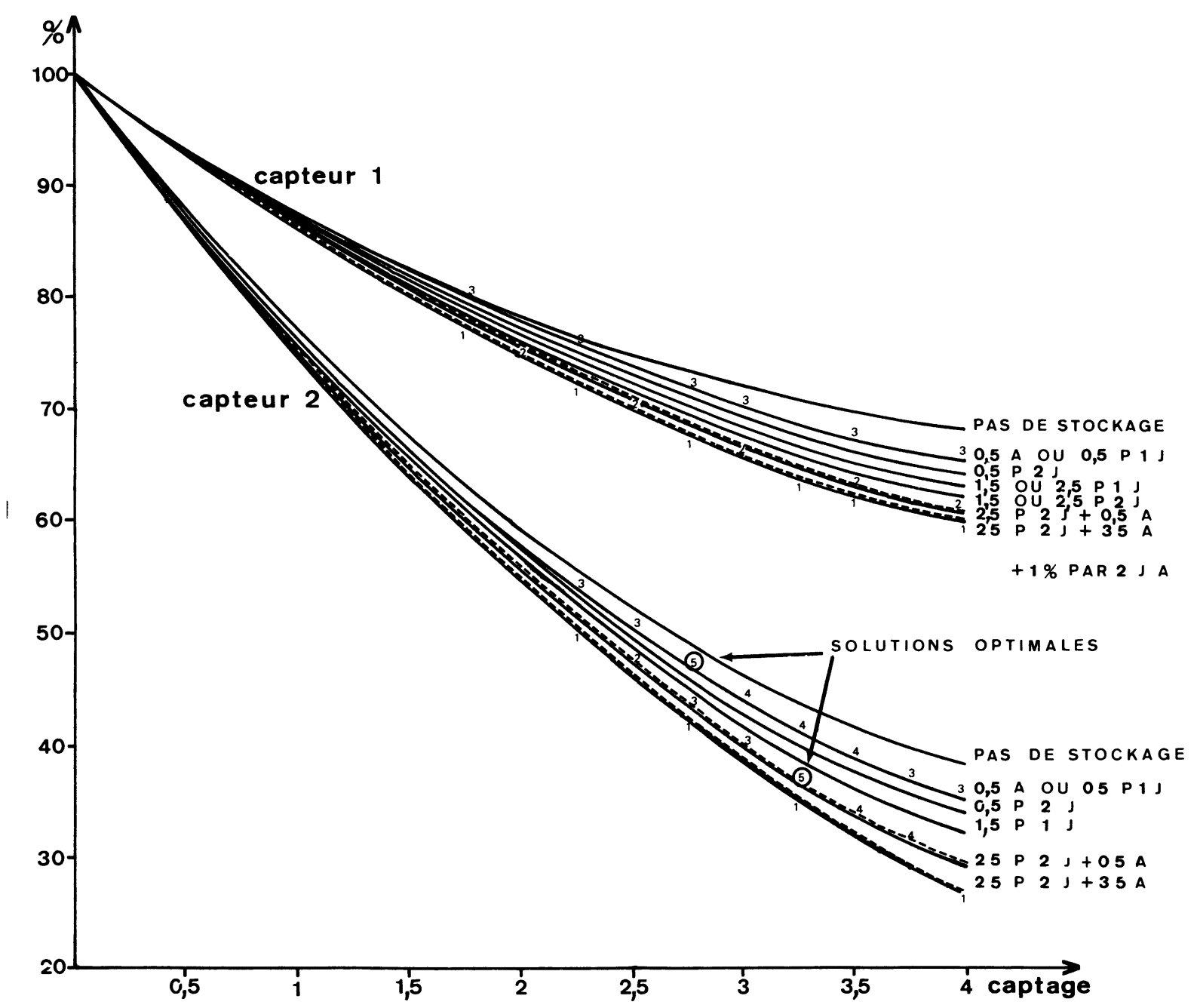

Fig. 8. - Région parisienne.

[Paris surroundings (drawing conventions same as in Fig. 4).]

naires, la seule modification à considérer étant la récupération des calories en fin de journées pour un abaissement de la température des locaux pendant la nuit. Si les besoins sont à $G$ équivalents moindres, les stratégies et possibilités de chauffage solaire restent les mêmes.

Pour les résidences occupées 2 jours sur 7, et pour lesquelles on tient à assurer un minimum de $5^{\circ}$ dans l'habitation pendant les 5 autres jours, on pourrait penser au premier abord qu'avec les mêmes installations la couverture des besoins sera plus grande que lorsque l'occupation est permanente. Ceci est faux pour les installations comprenant un stockage trop faible : en effet pour une journée de milieu d'hiver où il n'y a pas besoin de chauffer, on supprime aussi deux journées, une de début d'hiver et une autre de fin d'hiver ; or pour ces journées la couverture des besoins est plus grande que ne l'est celle pour l'hiver complet. D'où une diminution de la couverture des besoins.

L'analyse de la courbe 9 fait ressortir l'intérêt du captage jusqu'à une certaine limite, ainsi que l'intérêt du stockage, et en particulier du stockage annuel. Deux remarques sont à faire :
- le stockage journalier n'est pas du tout à négliger ;

— une couverture des besoins à $100 \%$ est difficile à atteindre. Pour s'en convaincre il suffit d'observer (Fig. 3) qu'il y a par hiver au moins une séquence de plus de 7 jours de suite de mauvais temps. Par conséquent un stockage annuel doit pouvoir assurer au moins 2 week-ends successifs et être rechargeable, pour moitié en moins de 5 jours, ou pouvoir assurer 3 week-ends (6 jours, soit environ $12 \%$ des besoins). Or un captage de $1 \mathrm{cs}$ avec un stockage de 6 cs est une surdimension, tout autant qu'un captage de $1,5 \mathrm{cs}$ et un stockage de $4 \mathrm{cs}$ : de telles installations ne sont utilisées à fond qu'au plus une fois dans l'hiver. On retrouve donc ici encore l'intérêt de l'appoint pour une limitation du captage et du stockage à moins de $1 \mathrm{cs}$. L'appoint peut être supprimé si l'on accepte de ne pas utiliser le logement par trop mauvais temps (c'est d'ailleurs presque toujours ce qui se fait).

Les figures 10 et 11 confirment ces mêmes observations pour les capteurs de type 2 à Nice, et ceux de types 1 et 2 à Paris. 


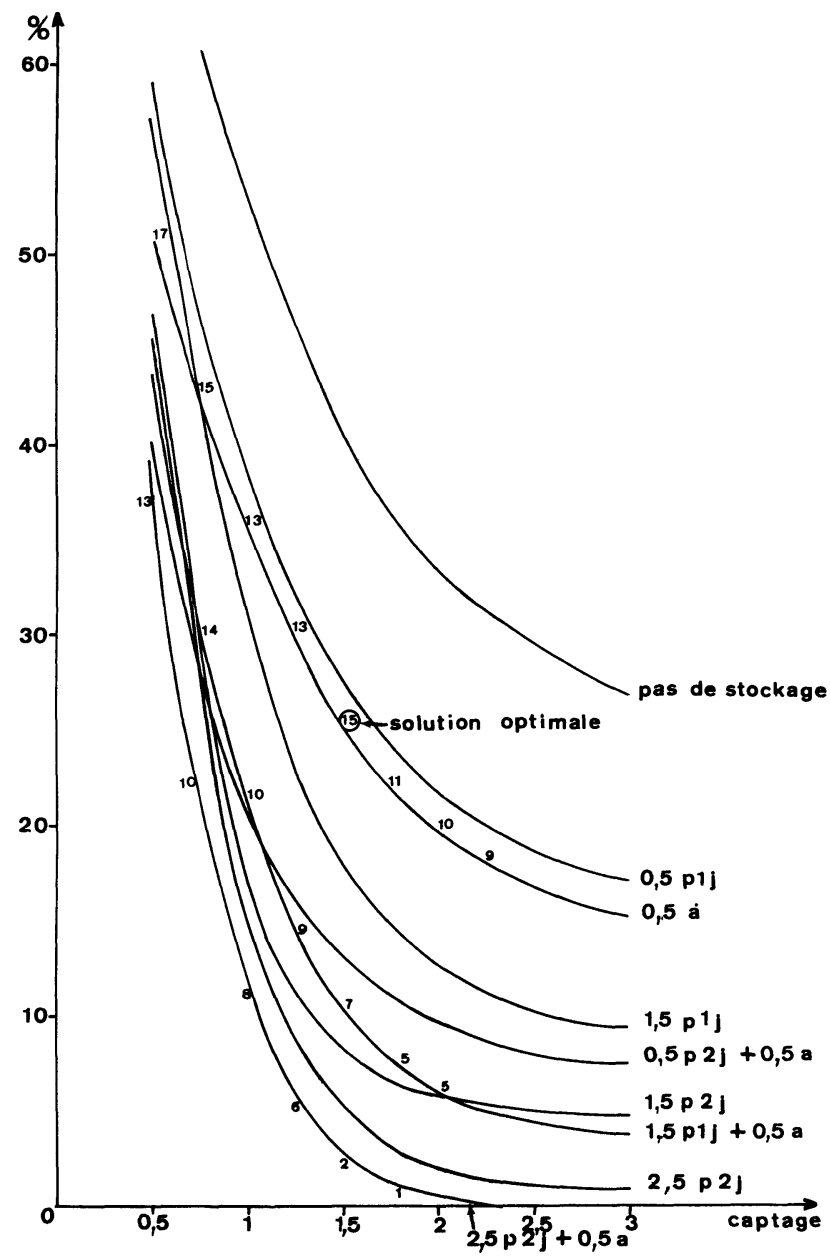

Fig. 9. - Nice (W. E.) - capteur 1.

[Nice - week-end - classical collector (drawing conventions same as in Fig. 4).]

9. Point de vue financier. - Dans la perspective d'un amortissement en 10 ans, un stockage rechargé 12 fois ne doit pas coûter plus de 120 fois la capacité cs associée. Si on admet que cs vaut environ $80 \mathrm{~kW}$ à $25^{\circ} \mathrm{C}$, on a un prix de $2400 \mathrm{~F}$ qui devrait couvrir $1 \mathrm{cs}$ de stockage et $0,5 \mathrm{cs}$ de captage, soit $15 \mathrm{~m}^{2}$ de capteur et $2 \mathrm{t}$ de chliarolithe. On est loin du compte. Il n'y a donc pas dans ce cas de rentabilité, mais seulement une assurance du même ordre que celle qui conduit à la majoration des capacités de chauffage. Le prix maxi de cette assurance peut être fixé à $30000 \mathrm{~F}$ par exemple et la stratégie reste alors dans des prix acceptables.

Il faut admettre que dans le captage-stockage une partie est rentabilisée parce qu'utilisée de nombreuses fois. Une autre partie ne l'est pas. Son seul intérêt est d'assurer la couverture des besoins.

De même, dans une maison de $130 \mathrm{~m}^{2}$, il y a environ une dizaine de radiateurs électriques de $0,75 \mathrm{~kW}$ à $1,5 \mathrm{~kW}$; or 4 ou 5 seulement sont en fonctionnement (et encore intermittent) la plupart du temps en hiver. Les autres ne sont utilisés que certains jours très froids.

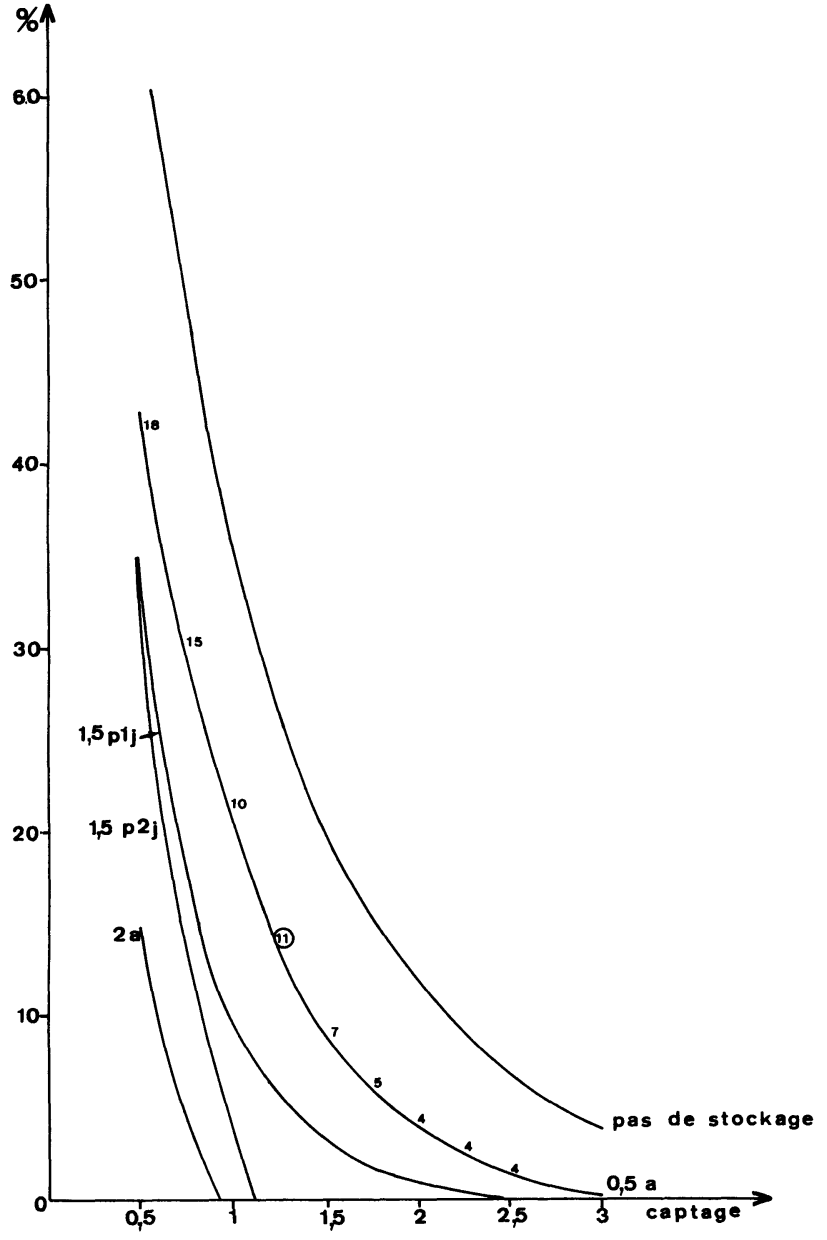

Fig. 10. - Nice (W. E.) - capteur 2.

[Nice - week-end - collectors of type 2 (drawing conventions same as in Fig. 4).]

10. Conclusion. - L'étude présente a voulu montrer comment le climat d'une région a une influence sur une installation de chauffage solaire, et préciser quel pourcentage limite de couverture des besoins il est possible d'atteindre. Si les capteurs sont des appareils coûteux, les baies vitrées sont par contre un moyen esthétique, efficace et peu onéreux pour capter l'énergie solaire; mais il ne faut pas en abuser : le confort dépend non seulement de la température de l'air des locaux, mais aussi du rayonnement qui peut être perçu : les rayonnements froids de grands vitrages et chauds des parois insolées, ainsi que le rayonnement solaire direct engendrent un inconfort certain. Sans développer ici ce problème du confort dans les espaces survitrés [8], il faut retenir qu'en région niçoise les apports directs associés à une inertie moyenne et un $G$ de l'ordre de 1 peuvent assurer près de $40 \%$ des besoins de chauffage mais seulement $10 \%$ en région parisienne.

Adopter un type d'installation solaire à une région et un habitat, c'est finalement tenir compte en même temps que des séquences de temps, des gammes dans lesquelles oscillent les températures et l'hygrométrie 


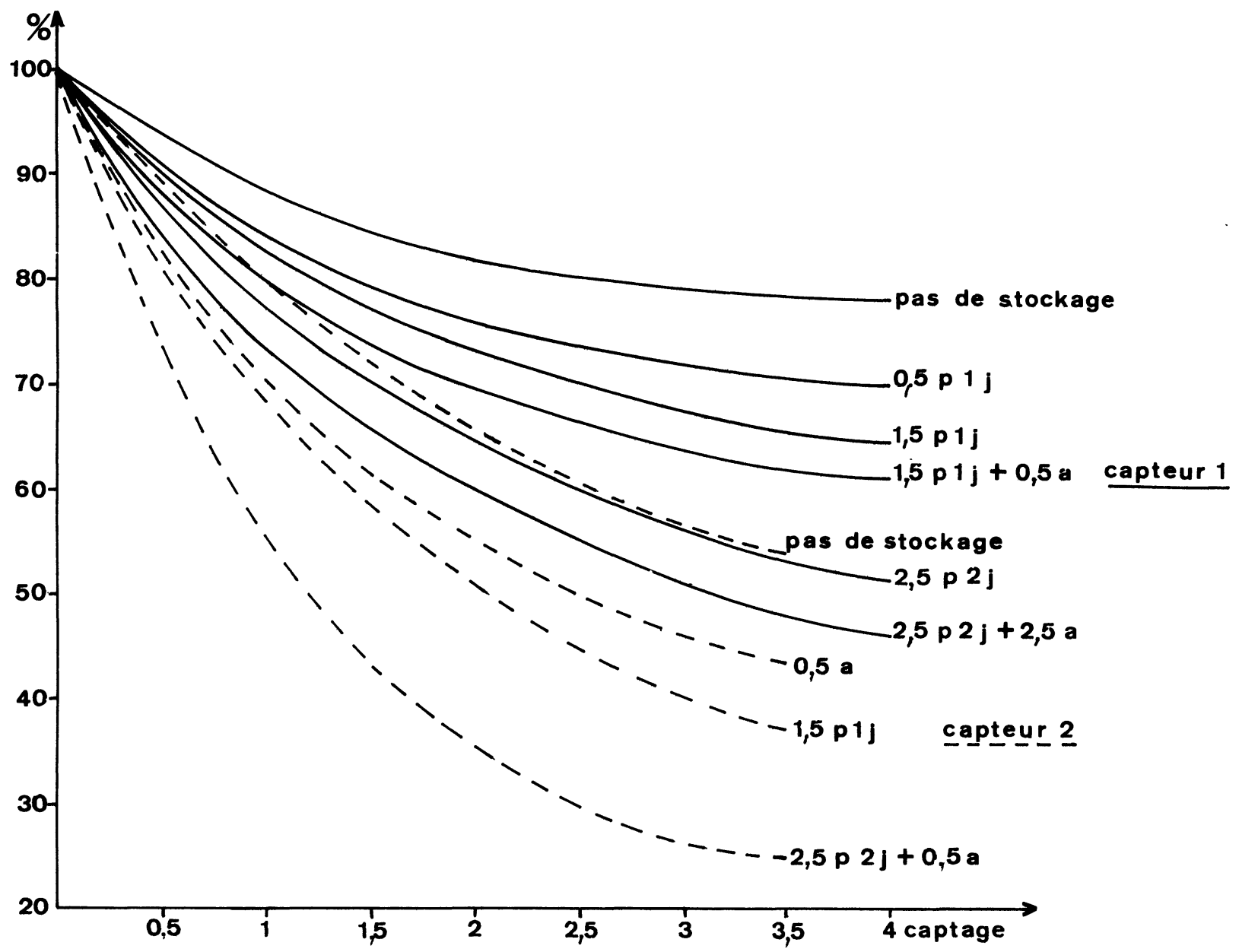

Fig. 11. - Paris (W. E.).

[Paris surroundings - week-end (drawing conventions same as in Fig. 4).]

de l'air extérieur, des situations extrêmes qui se présentent dans le climat, de l'usage qui doit être fait de l'habitat. Une installation doit être une optimisation et non une réalisation libre dans sa conception. Les stratégies d'utilisation de l'appoint ne sont pas compliquées et on ne peut en attendre une augmentation du taux de couverture des besoins de plus de 7 à $8 \%$.

\section{Bibliographie}

[1] Schneider, M., Berger, X., Bourdeau, L., Jaffrin, A., SylVAIN, J. D., Conception de chauffage solaire en climat méditerranéen, colloque Liège (1977).

[2] Peube, J. L., Pécheux, J., Planco, E., Sur les conditions optimales d'utilisation de l'énergie solaire pour le chauffage de l'habitat, Revue Phys. Appl. 15 (1980) 553.

[3] Berger, X., Etude du climat en région niçoise en vue d'applications à l'habitat solaire, extrait de Analyse statistique des processus météorologiques appliquée à l'énergie solaire, publication C.N.R.S. (1979).

[4] BERGER, X., Serres accolées à l'habitat, stores vénitiens à chaleur latente, Rapport Plan Construction « maison Energie Zéro » (1980).
[5] Clément, R., Berger, X., La maison solaire de Mougins, colloque M.I.S. Nice (1980).

[6] Olivet, J., Hamayon, L., Cabinet Teta, Groupe Ecothermique Solaire, Cabinet Parica T.S.I., optimisation d'un modèle d'habitat à double façade ventilée, Rapport Plan Construction (1980).

[7] Schneider, M., Petitcollot, A. M., Berger, X., Ray, P., Association of heat pumps with trickle unglazed solar collectors, colloque Stockholm (1980)

[8] SCHNeIDER, M., BerGer, X., Etude d'ambiances de rayonnement dans les systèmes solaires passifs, colloque M.I.S. Nice (1980). 